\title{
Purification of Chloromethane by Selective Adsorption of Dimethyl Ether on Microporous Coordination Polymers
}

\author{
Jake A. Boissonnault, Antek G. Wong-Foy, Adam J. Matzger*
}

*Department of Chemistry, Macromolecular Science and Engineering Program, University of Michigan, 930 N.

University Avenue, Ann Arbor, Michigan 48109, Email: matzger@umich.edu

\section{Supporting Information}

I. MCP Analyses

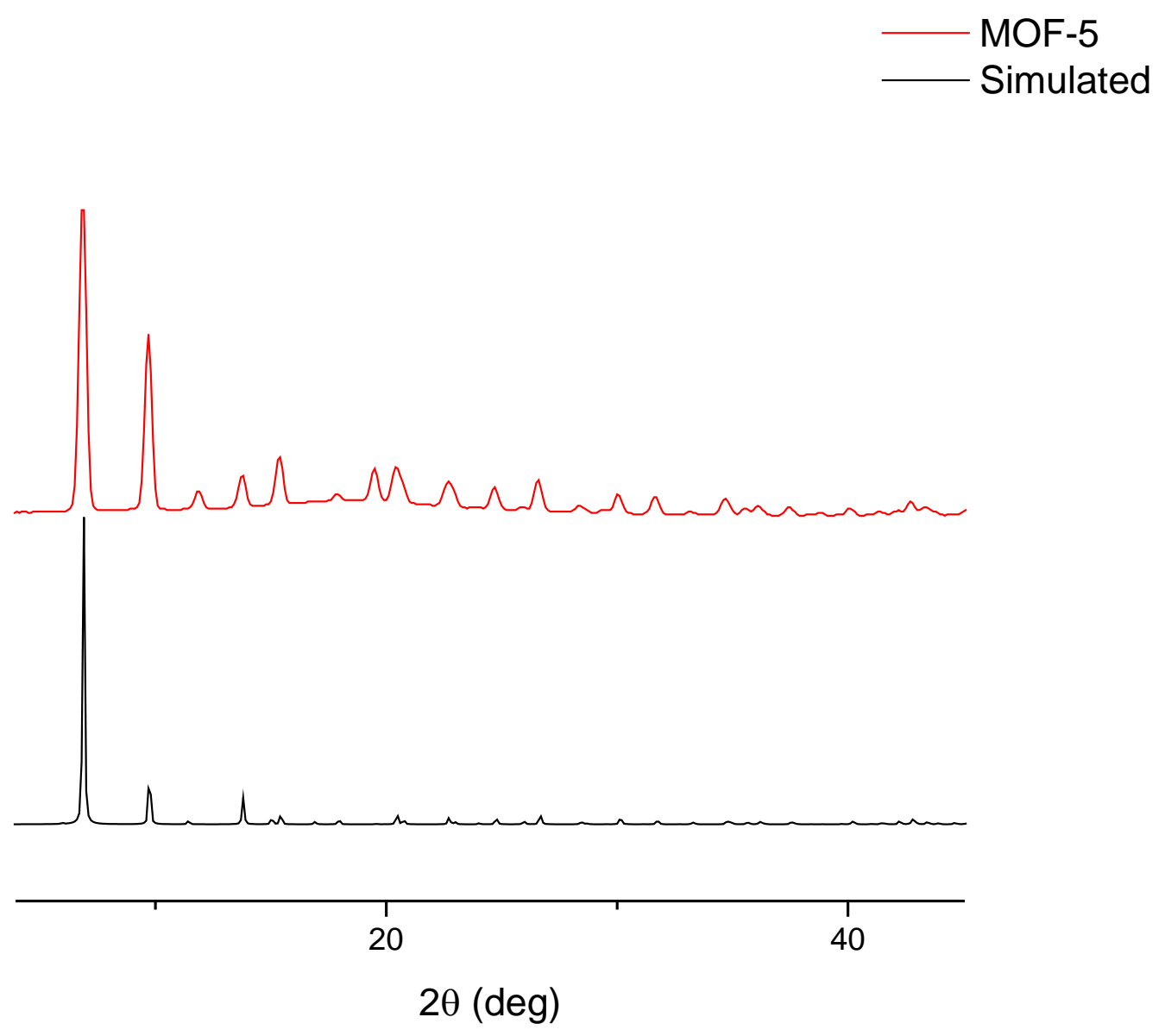

Figure S1. MOF-5 powder x-ray diffraction pattern. 
Co/DOBDC

Simulated

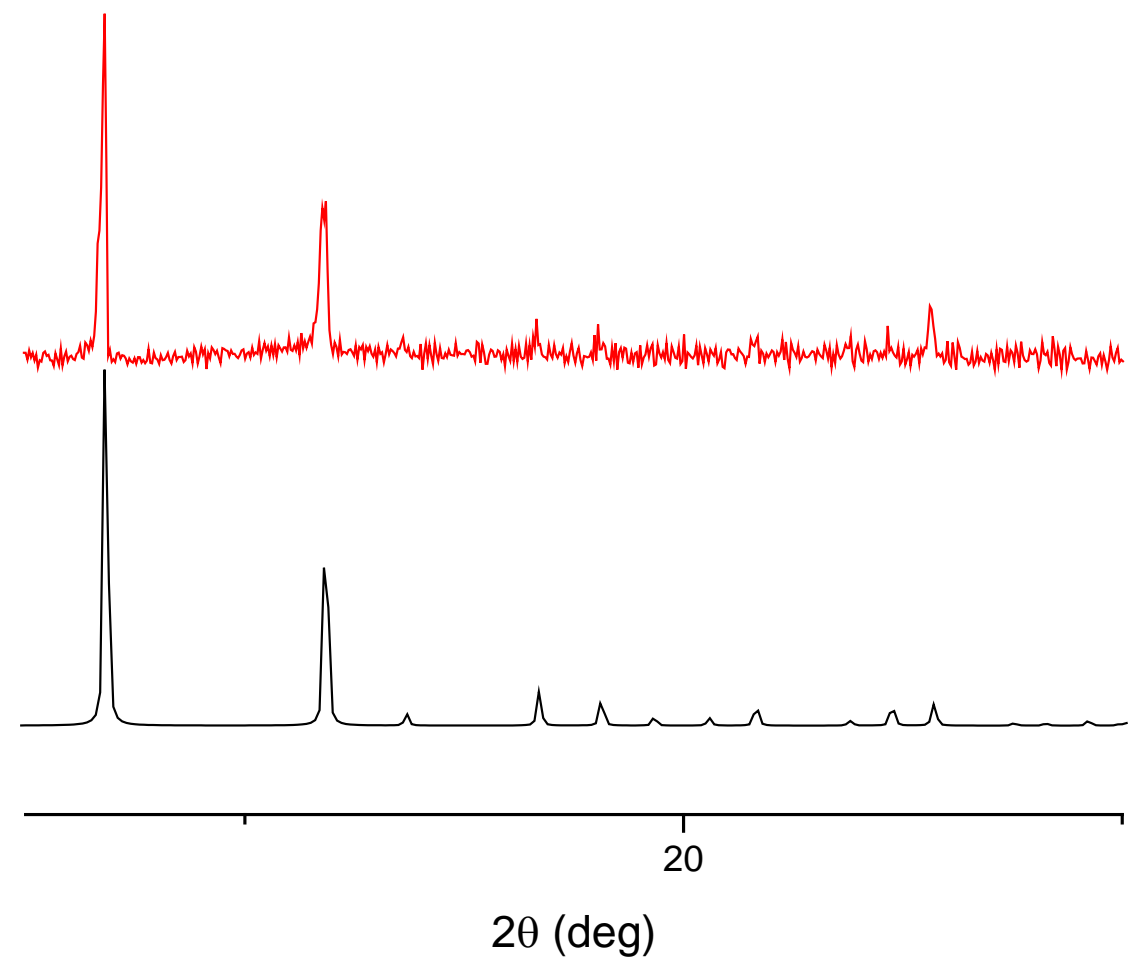

Figure S2. Co/DOBDC powder x-ray diffraction pattern. 
MIL-100(Fe) Simulated

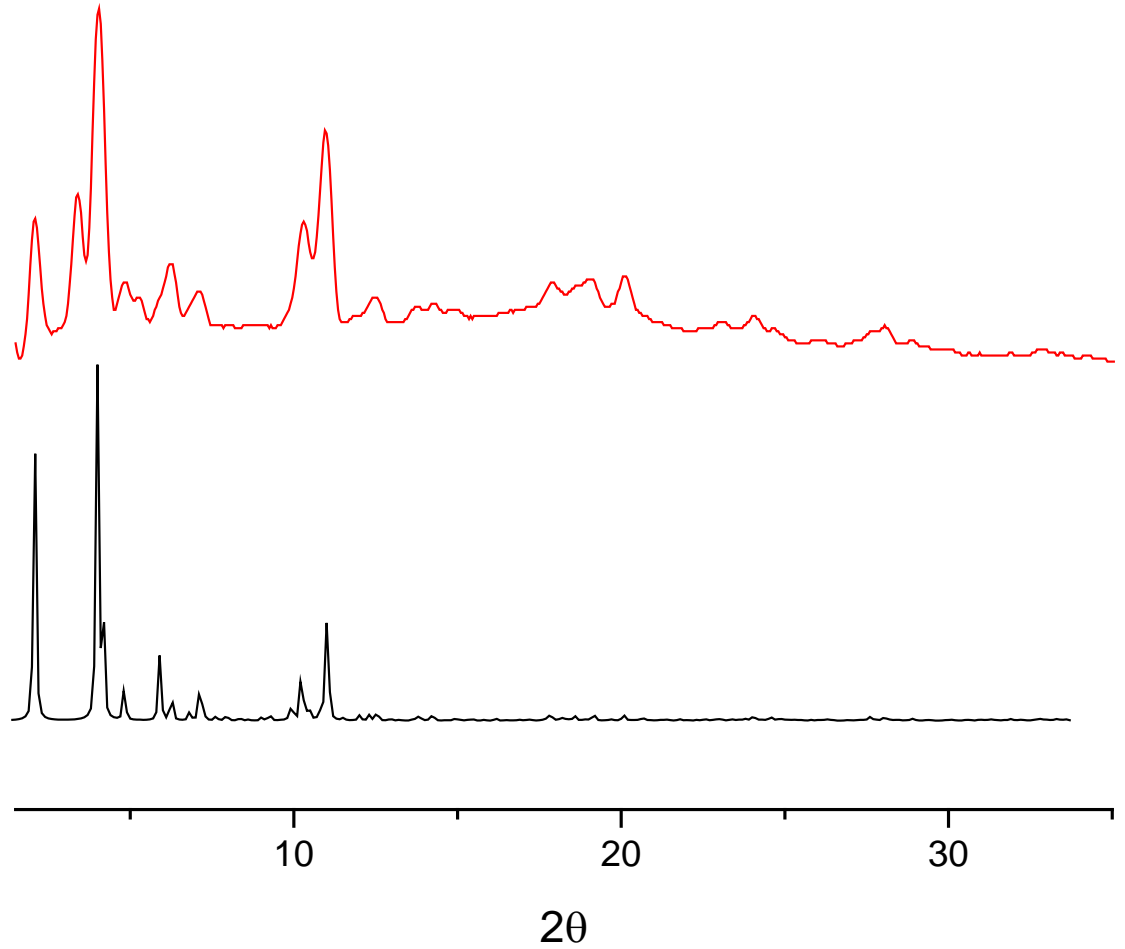

Figure S3. MIL-100(Fe) powder x-ray diffraction pattern. 
HKUST-1

Simulated

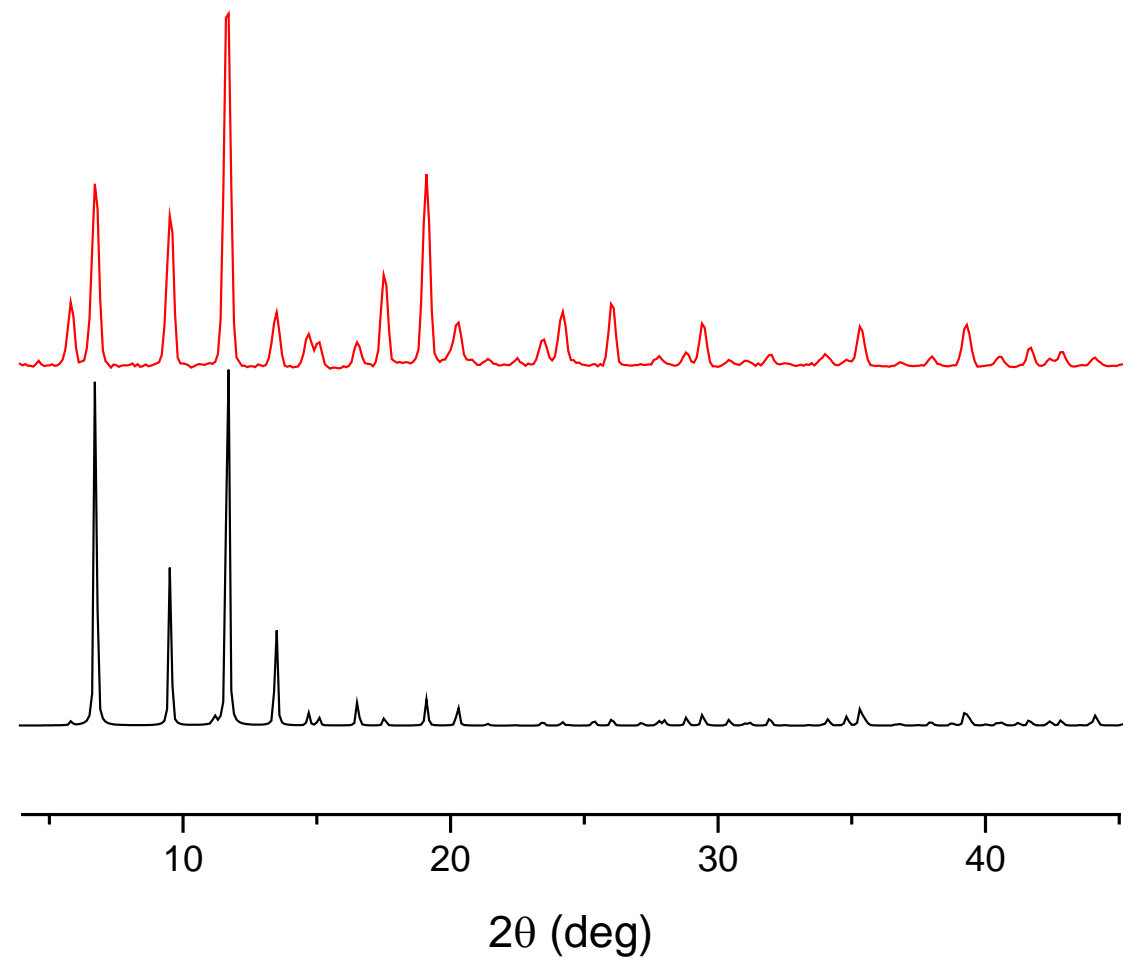

Figure S4. HKUST-1 powder x-ray diffraction pattern.

\begin{tabular}{|c|c|}
\hline Sample & BET surface area $\left(\mathrm{m}^{2} / \mathrm{g}\right)$ \\
\hline MOF-5 & 3032 \\
\hline MIL-100(Fe) & 2176 \\
\hline HKUST-1 & 1188 \\
\hline Co/DOBDC & 1146 \\
\hline
\end{tabular}

Table S1. BET surface area calculation of the MCPs used for the breakthrough experiments. 


\section{Breakthrough Apparatus}

A diagram of the apparatus is shown below in Figure S1. Sorbent material ( $30 \mathrm{mg}$ ) prepared above was loaded into a glass tube (O.D. 1/4", I.D. $0.148^{\prime \prime}$ ) supported by glass wool on either end under an inert atmosphere. The tube (1) was placed in line with the flow system under a flow of nitrogen gas. Chloromethane (@49 sccm, 2) and dimethyl ether (@1 sccm, 3) gases were mixed in line prior to flow over the sorbent. The flow rate of the gas mixture was adjusted to $10 \mathrm{sccm}$ using an MFC and backpressure valve (4) before flowing through the sorbent. The effluent from the system was measured through a residual gas analyzer (5) mass spectrometer observing peaks at 28,46 and 51 mass units to observe dinitrogen, $\mathrm{DME}$ and $\mathrm{MeCl}$ respectively.

The switch at (6) allowed for the sorbent packed into a glass tube to be kept under nitrogen flow until the experiment was started by flipping the switch to the MeCl/DME mixture. Due to this, there is a dead volume in the system corresponding to the volume that the gas mixture has to fill before it can be seen by the detector. The dead volume of the system was calculated by switching the gas mixture with a column packed with an amount of glass wool similar to that used to hold MCP and measuring the amount of time it took for MeCl/DME to be detectable by the mass spectrometer. This gave a calculated dead volume of $2.21 \pm 0.05 \mathrm{~mL}$ which is further subtracted from all of the results as described below. 


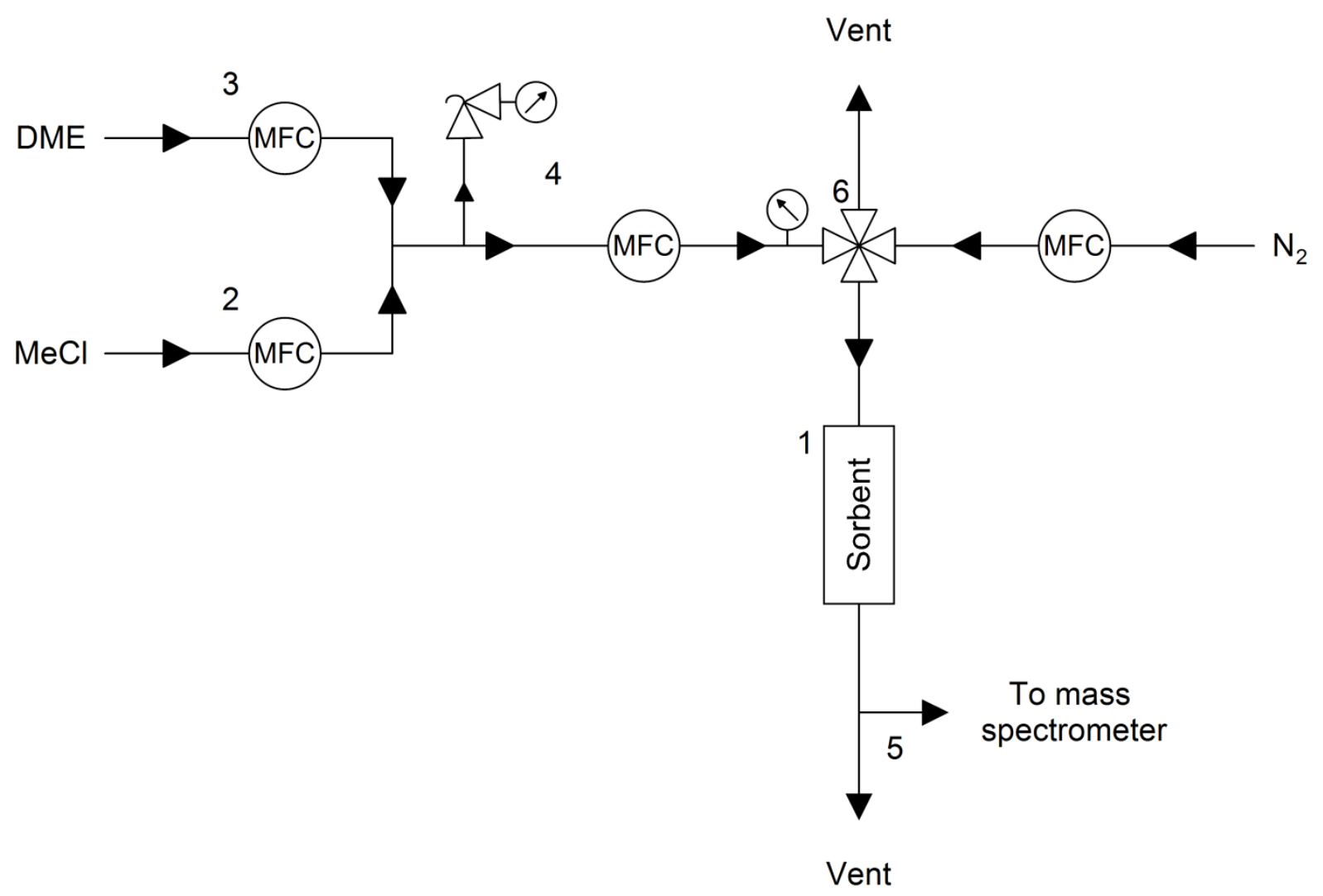

Figure S5. Block flow diagram of apparatus.

\section{Breakthrough Analysis Methods}

Mass spectrometry data are collected at a rate of $3 \mathrm{~Hz}$ with the components given as a partial pressure (torr). Breakthrough experiments were allowed to proceed until the mixture had achieved equilibrium for both gases.

The data is converted to volume of gas by first determining a flow factor that corresponds to the signal of the gas at the maximum in the flow system (ie. $98 \%$ for $\mathrm{MeCl}$ and $2 \%$ for DME). The flow factor is determined by:

$$
f f_{\text {gas }}=\frac{\text { flow rate }_{\text {gas }}}{s_{e q}}
$$


where $f f$ is the flow factor in $\mathrm{mL} /(\mathrm{s} \cdot$ torr), flow rate is given as $\mathrm{mL} / \mathrm{s}$ for that gas as a part of the mixture, and $s_{e q}$ is the signal of the gas at equilibrium given in torr. From this, the volumetric flow rate can be determined by multiplying $f f$ by the signal at each time point. This can then be used to determine the volume of gas that has flowed into the system over each separate time point A and A-1 using the trapezoidal approximation:

$$
V o l_{A}=\left(f f *\left(s_{A}+s_{A-1}\right)\right) * 0.5 *\left(t_{A}-t_{A-1}\right)
$$

where $s_{\mathrm{A}}$ is the signal at time point $\mathrm{A}, s_{\mathrm{A}-1}$ is the signal of the time point directly before time point $\mathrm{A}$, and $t_{\mathrm{A}}$ and $t_{\mathrm{A}-1}$ are those respective times in seconds. The total capacity of gas adsorbed by the sorbent in each run is given by the theoretical amount of gas flowed over the system less the dead volume of the system and the sum of the gas detected at each point calculated as above:

$$
\text { Vol }_{\text {total }}=\text { flow rate } * t_{e q}-\text { Dead Volume }-\sum_{A}^{\text {teq }} \operatorname{Vol}_{A}
$$

with $t_{\text {eq }}$ being the time until equilibrium is reached. Each volume adsorbed was averaged across three separate runs for each material.

Volume of pure $\mathrm{MeCl}$ obtained from each sample is determined by calculating the amount of $\mathrm{MeCl}$ detected before the appearance of DME in the mass spectrometer. This is done by subtracting the amount of $\mathrm{MeCl}$ adsorbed to the MCP from the total amount of $\mathrm{MeCl}$ that flows through the system before detection of DME as:

$$
\text { Pure MeCl }=\text { flow rate } * t_{D M E, b k t h}-\text { Dead Volume }-\sum_{A}^{t_{M e c l, e q}} \operatorname{Vol}_{M e C l, A}
$$

where $t_{b k t h}$ is the time of breakthrough of the gas component when it is detected by the mass spectrometer. The volume of pure $\mathrm{MeCl}$ is calculated separately for each run and averaged for each material. 


\section{Regeneration Studies of Co/DOBDC}

A sample Co/DOBDC subjected to the above gas mixture stream was regenerated in its glass column on the system using a heating jacket. The material was heated to an internal temperature of $145^{\circ} \mathrm{C}$ under nitrogen flowing at a continual rate of $20 \mathrm{sccm}$. The effluent was monitored by RGA mass spectrometry and the sample was heated until no more DME was observed. The column was then allowed to cool under flowing nitrogen until it reached ambient temperature. The material was then subjected to the $\mathrm{MeCl} / \mathrm{DME}$ stream as above to determine any differences in the separation properties. Regeneration cycles were monitored by the mass spectrometer at a rate of $0.3 \mathrm{~Hz}$.

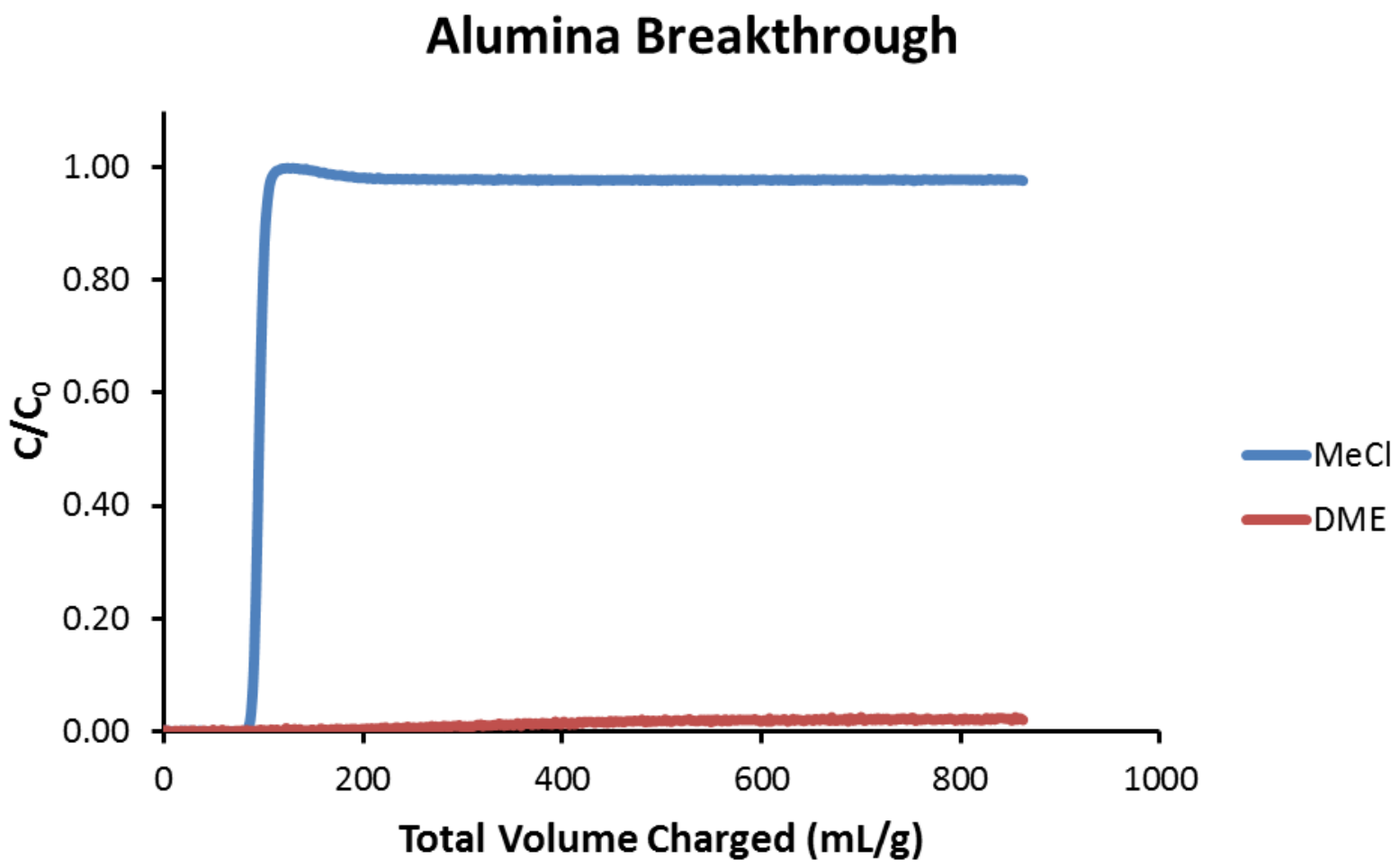

Figure S6. DME/MeCl breakthrough curve of activated alumina. 


\section{Davisil Grade 923 Breakthrough}

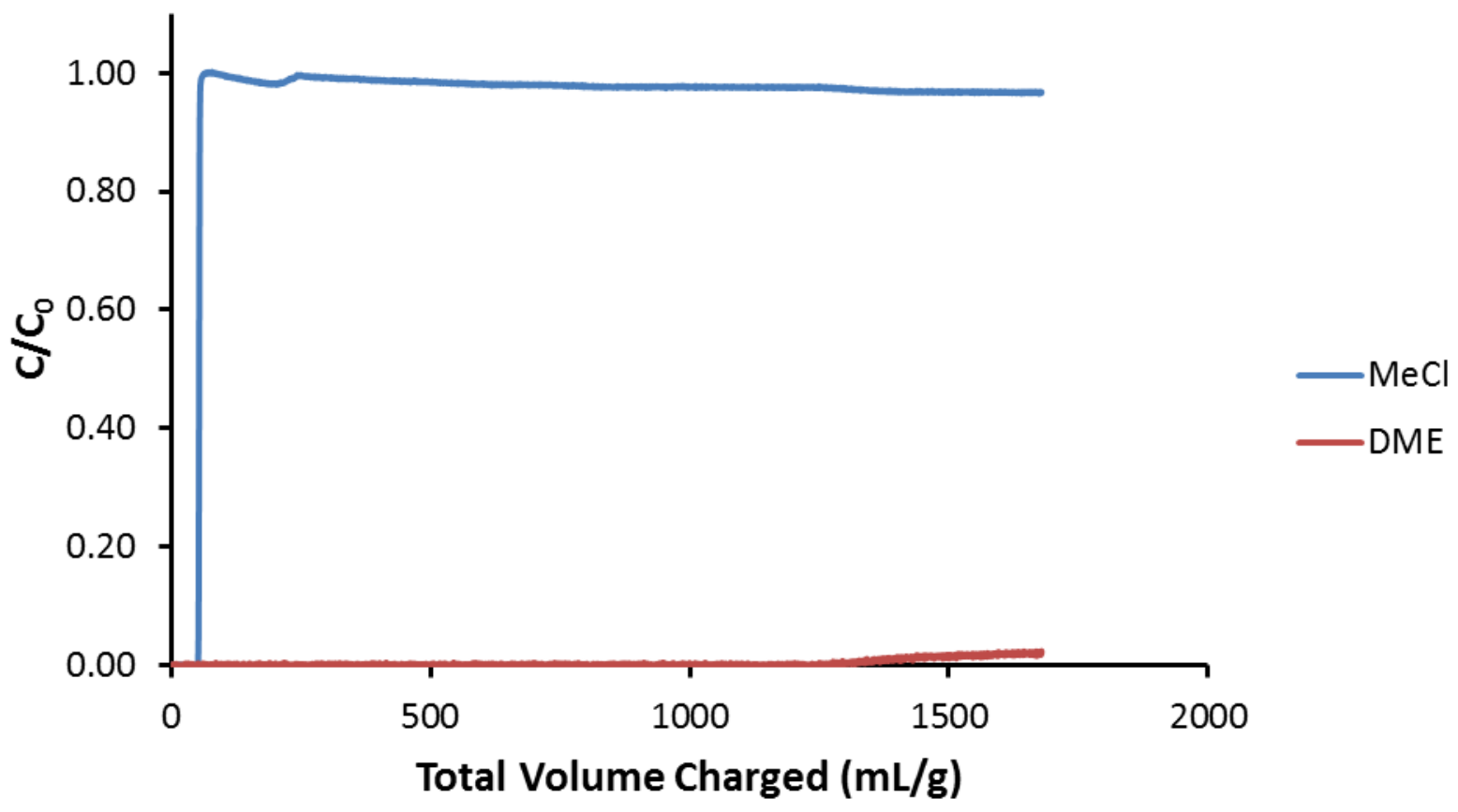

Figure S7. DME/MeCl breakthrough curve of Davisil grade 923. 


\section{Davisil Grade 635 Breakthrough}

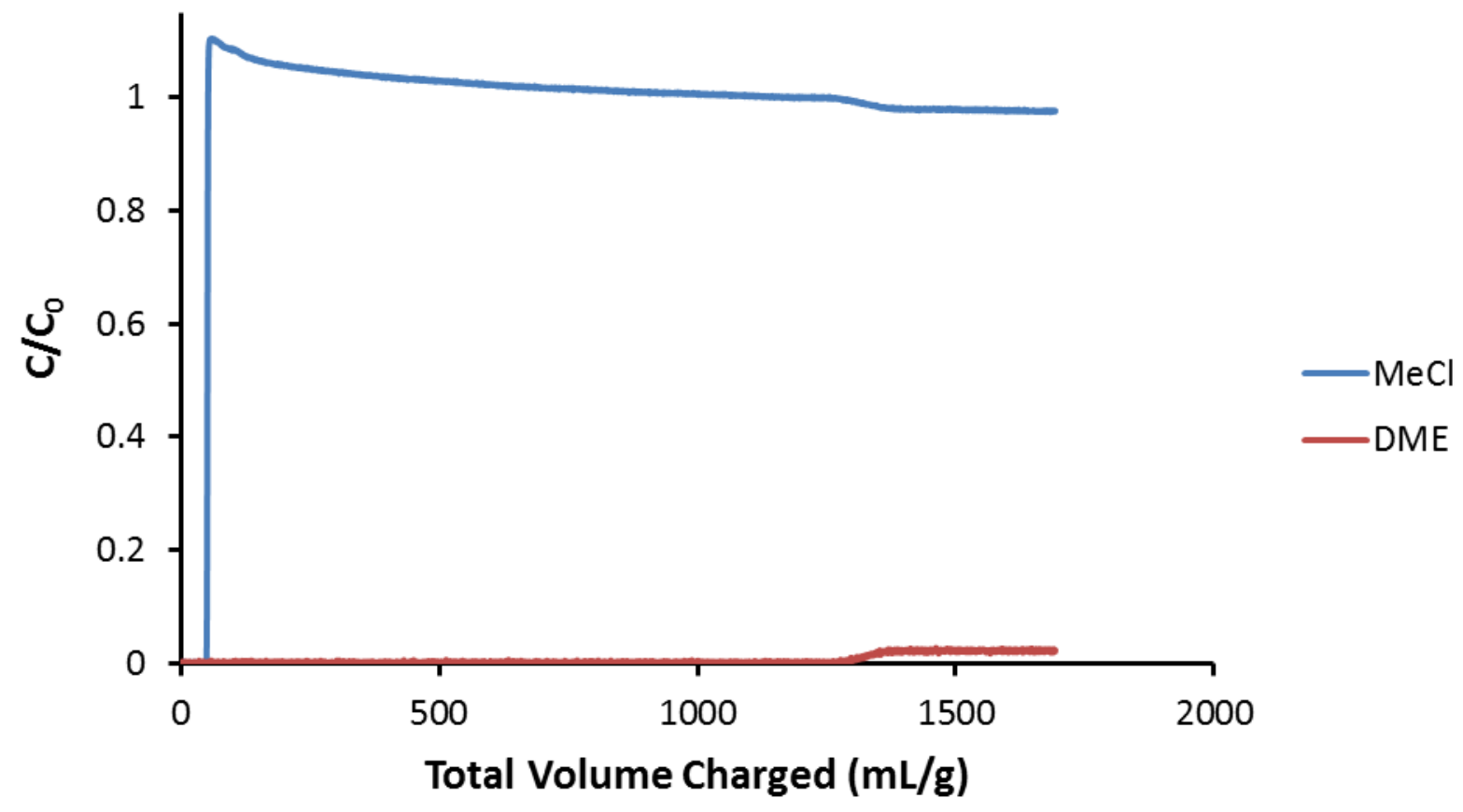

Figure S8. DME/MeCl breakthrough curve of Davisil grade 635 . 


\section{Co/DOBDC Breakthrough}

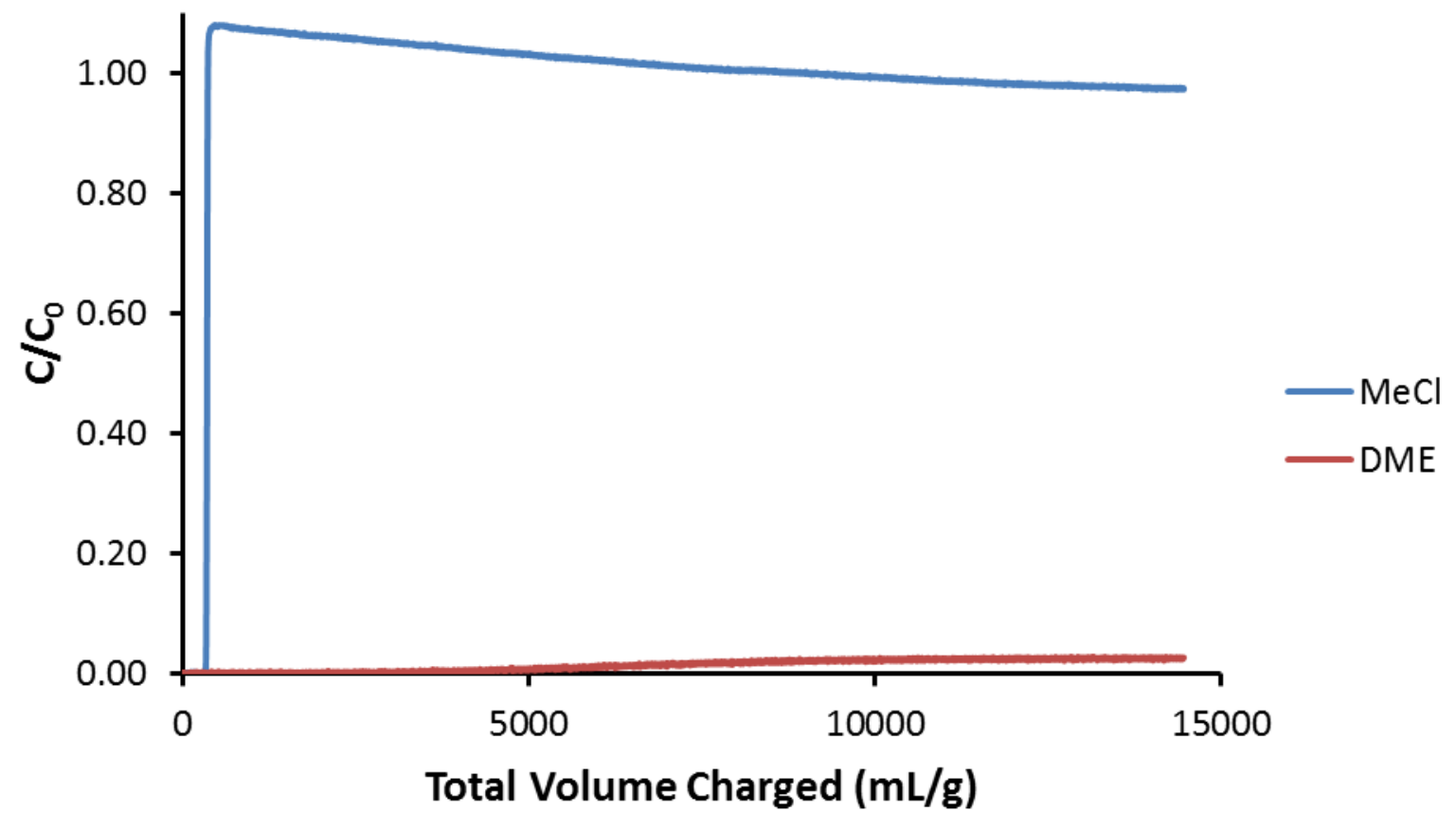

Figure S9. DME/MeCl breakthrough curve of Co/DOBDC. 


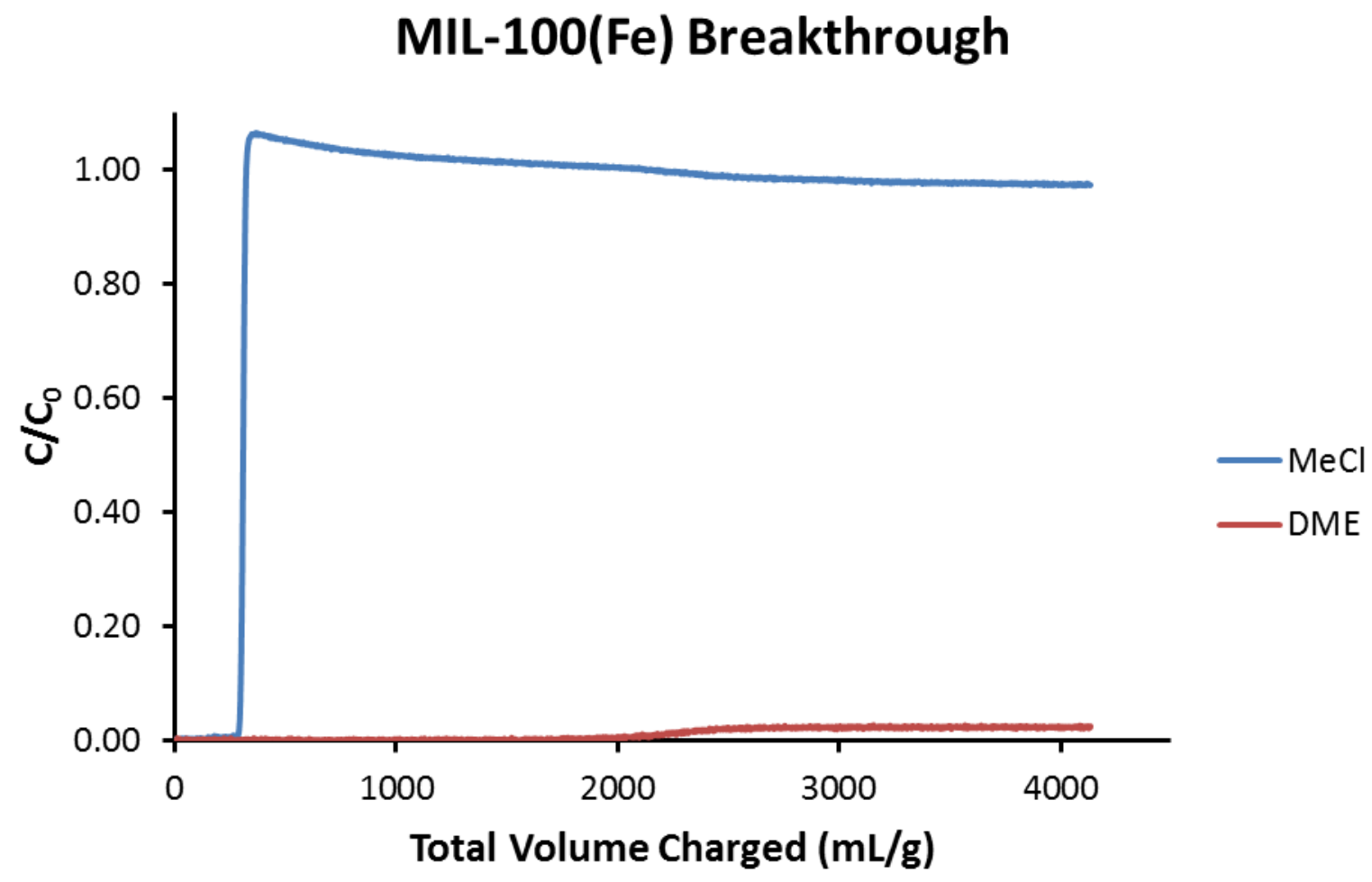

Figure S10. DME/MeCl breakthrough curve of MIL-100(Fe). 


\section{HKUST-1 Breakthrough}

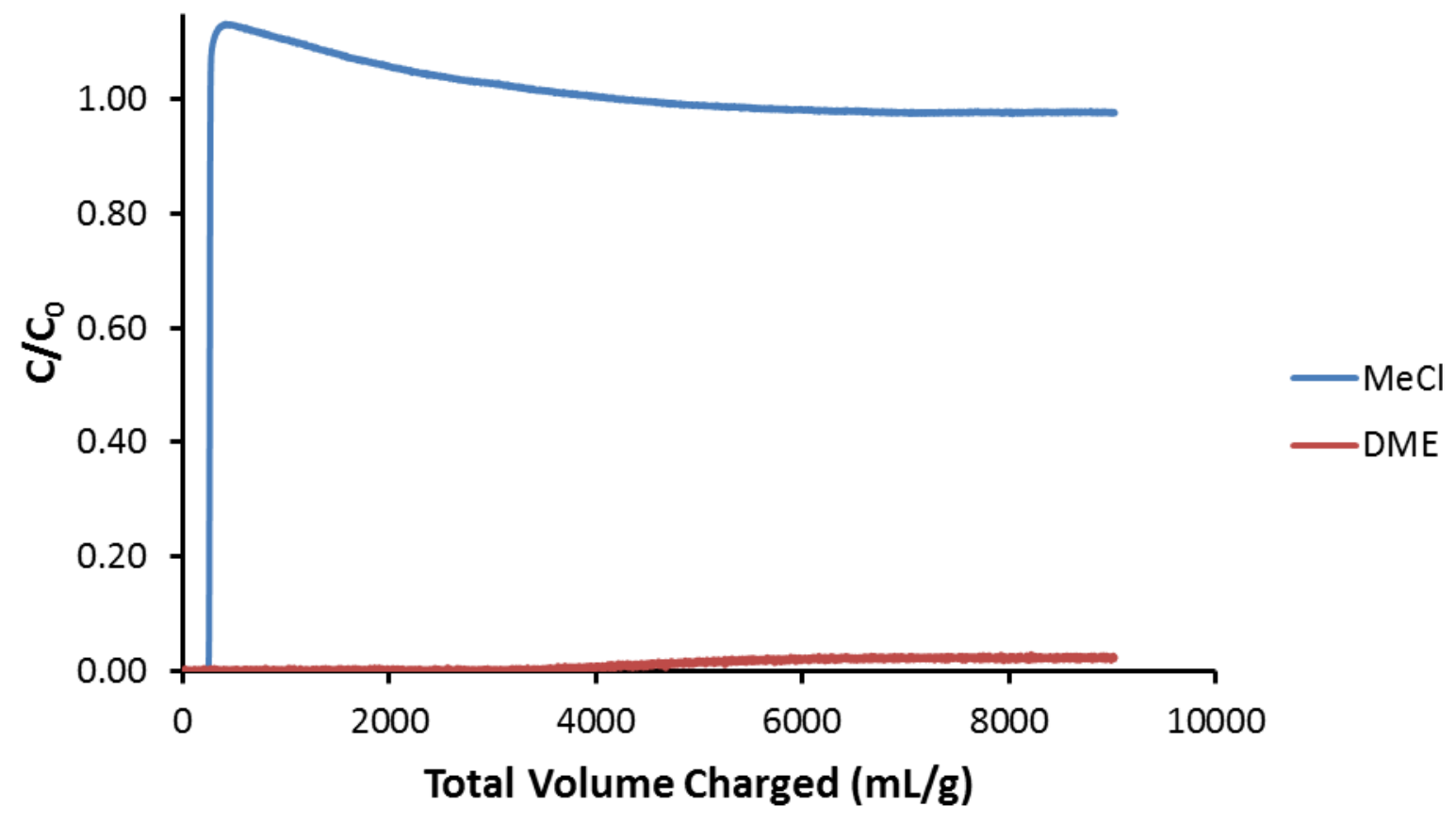

Figure S11. DME/MeCl breakthrough curve of HKUST-1. 
MOF-5 Breakthrough

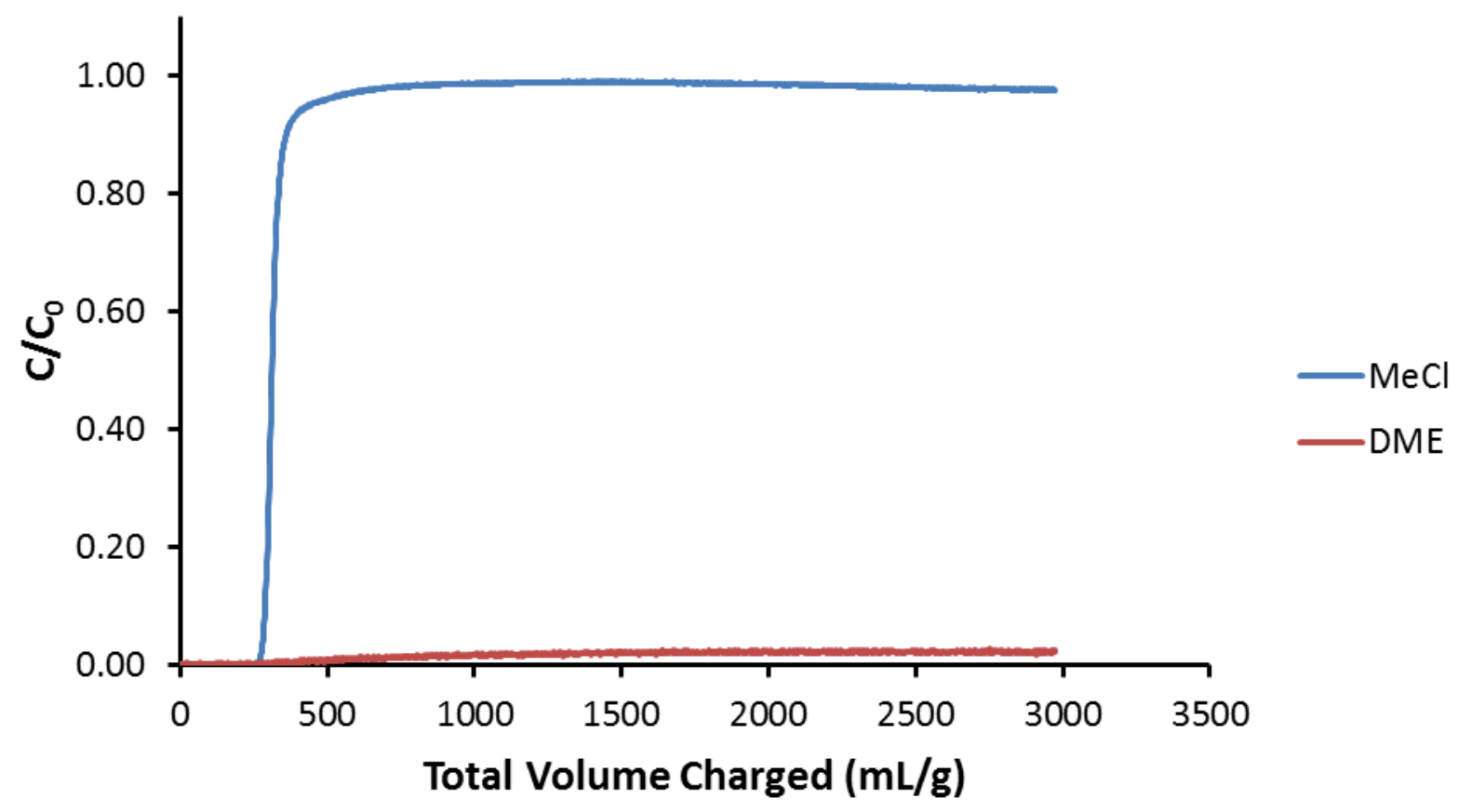

Figure S12. DME/MeCl breakthrough curve of MOF-5. 